\title{
Development of Self-healing Coatings for Corrosion Protection on Metallic Structures
}

\author{
Alicja Stankiewicz*, Michael B Barker \\ School of Engineering \& the Built Environment, Edinburgh Napier University, 10 Colinton \\ Road, Edinburgh, EH10 5DT, United Kingdom \\ *corresponding author: a.stankiewicz@napier.ac.uk
}

\begin{abstract}
Inspired by biological systems, artificial self-healing materials are designed for repairing local damage caused by external factors. The rapidly expanding field of selfhealing systems contains, among others, materials with well-defined surface properties. Undoubtedly, enhancing surface functionalisation, by applying smart coatings, enjoys an extensive interest. The self-healing ability is particularly essential property for corrosion protection strategies, especially when the use of one of the most effective corrosion systems, based on chromium(VI) compounds, is now banned by the current REACH (Registration, Evaluation, Authorisation and Restriction of Chemicals) legislation. Self-healing protective coatings are produced using macromolecular compounds, ceramics, metals and composites. Considering the wide range of available materials, the number of potential combinations seems to be unlimited. The self-healing action of such coatings is activated by appropriate stimuli: temperature changes, radiation, $\mathrm{pH}$ changes, pressure changes and mechanical action.

In this paper, the research and practical implications of the various approaches to achieving self-healing functionality of protective coatings, as well as potential developments in this area, are explored.
\end{abstract}

Keywords: self-healing coating; protective coating; corrosion 


\section{Introduction}

In selecting a construction material one has to face a compromise between the desired properties and price. Very often the somewhat inadequate surface properties of magnesium and aluminium alloys, steel and composites, can be enhanced by covering their surface with a protective coating. This approach, apart from saving the costs of using more expensive materials, imparts desired properties such as hardness or abrasion resistance. Preventing and mitigating corrosion issues of structural and functional materials are a vast cost to modern industrialized economies. According to data published by the Corrosion Committee of the Surface Engineering Division (the Institute of Materials, Minerals and Mining (IOM3, UK), annual costs connected with chemical degradation or electrochemical corrosion to the UK economy are estimated between 2-3\% of Gross National Product (GNP). A similar situation is observed in USA and Japan [1]. More specifically it can be said, that corrosion costs around $£ 600$ per capita per annum. Often in these figures, the costs related to equipment idle time are highlighted. However, replacing, repairing or restoring corroded equipment also requires substantial additional manpower and investment in new capital equipment. Nowadays, protective coatings have to meet the requirements of the current legislation embodied in the Registration, Evaluation, Authorisation and Restriction of Chemicals $(\mathrm{REACH})$, which prevents the use of harmful substances including hexavalent chromium, the main compound employed in such coatings until recently. Furthermore, the surface finishing sector is aware that it is not only the manufacture of the product, but also its use in service and its disposal at end-of-life that can impact the environment detrimentally. Consequently, there is a pressing need to render production methods less environmentally damaging without compromising on the product life cycle. The circumstances mentioned above have become the impulse for the development of alternative solutions [2]. Currently, coatings should not only fulfil requirements mentioned above but should also be easily manufactured, demonstrate reduced material usage and installation costs and exhibit enhanced durability. Therefore a strong emphasis is given by academic institutions as well as industry sector, to investigating and developing self-healing and smart coatings, combining various functionalities for improved corrosion protection. 


\section{Literature data}

Scientists are intensively trying to mimic natural processes and incorporate them into engineering materials. According to the data provided by Scopus, one of the largest abstract and citation databases of peer-reviewed literature, there have been 542 articles on self-healing protective coatings published between 1984 and November 2015. In fact, as fig.1 demonstrates, the growth in the number of articles was virtually exponential until 2014. However there is a slight downward trend this year with 61 articles published by November 2015 some 24 fewer than at the same time in 2014 .

The first publications describing self-healing protective coatings appeared in the middle of the 1980s. However those articles dealt with coatings containing carcinogenic hexavalent chromium. The self-healing action of a chromium-containing coating was based on the formation of a protective oxide film in the damage area. The presence of $\mathrm{Cr}(\mathrm{VI})$ provides a self-repair mechanism by reduction to $\mathrm{Cr}(\mathrm{III})$ during oxidative attack. A breakthrough in self-healing coatings' technology was the work of S. R. White et al. The authors presented the results of research on a polymer coating with self-repair functionality resulting from the action of polymerizing agents loaded into microcapsules [3]. Besides a microencapsulated healing agent, a catalytic chemical trigger was incorporated within an epoxy matrix. Embedded microcapsules were ruptured mechanically and released the healing agent into the crack area through capillary action. The polymerisation of the healing agent was then initiated by the action of the catalyst in the epoxy coating which subsequently led to the bonding of the crack faces. Additionally a healing concept of using living polymerization catalysts -without terminated chain-ends, enabled repeated healing activity.

The breakdown of research into self-healing coatings on a global basis is shown in fig. 2 and 3 . Most of the publications concerning self-healing coatings for protection against corrosion, describe the use of polymers and composites contained within the coatings. Generally, self-healing protective coatings can be divided into two types: self-healing polymeric coatings and self-healing non-polymeric coatings (inorganic coatings). However, it is also reasonable to classify them according to the coatings' functionality. Both types of coatings, for example, can prevent corrosion by releasing an active substance from capsules inserted into them. Taking this into account, coatings can be classified as follows:

- polymeric coatings; 
- coatings containing capsules;

- hybrid-oxide coatings;

- other self-healing coatings including conversion coatings, metallic coatings, titania coatings with casein and coatings containing ceramic materials.

\subsection{Polymeric coatings}

The literature provides many descriptions of polymeric coatings including those containing thermoplastic polymers, thermoset polymers, elastomers, shape memory polymers, supramolecular polymers, polymer composites and nanocomposites or biopolymers as a matrix [4-14]. The healing mechanism in polymeric materials is mainly based on the reversibility of a specific structural element. To achieve this effect, reversible covalent bonds (e.g. based on the Diels-Alder reaction) or weaker noncovalent interactions (e.g. hydrogen bonding, ionic interactions, $\pi-\pi$ interactions, or host-guest interactions) have been effectively employed [15-20].

Different kinds of polymer-based materials have been used successfully for a number of applications. This is a consequence of their inherent polymeric properties such as light weight; abundant availability; flexibility; ease of processing; and the ability to engineer the material to suit a given purpose.

In addition to the advantages of employing polymers as a coating matrix, other attributes can be added, notably:

- the ease of application (high temperatures are not required to form the coatings);

- the ease of modification (protective properties can be derived from various chemical and physical processes, e.g. oxidation, swelling or cross-linking);

- the possibility of inserting functional additives like corrosion inhibitors.

Depending on the polymeric coating employed, self-healing activity is achieved by different mechanisms. Specific examples of these processes are presented in Table 1. In systems containing conductive polymers, a self-healing process operates when a polymer is in its electroactive state [23]. For example, using an electroactive polyaniline emeraldine salt (ES) induces oxide growth on aluminium and its alloys and on iron surfaces. The acidity of cations of metal oxides has to be taken into account, as less acidic cations, e.g. $\mathrm{Zn}^{2+}$ or $\mathrm{Mg}^{2+}$, are unstable in contact with ES and depronate ES to electroinactive polyaniline emeraldine base (EB) [24]. 
The incorporation of a self-healing capability will clearly influence the appeal of utilising polymeric coatings. For example, in a porous polymer film of cellulose acetate with sodium benzoate as a corrosion inhibitor, polarization resistance is much higher than in a plain coating and, as a consequence, its durability is prolonged by at least three times [29]. Moreover, aluminium samples coated by two poly(ethyleneimine)/poly(styrene sulfonate) (PEI/PSS) bilayers do not exhibit any visible signs of corrosive attack even after 21 days immersed in a salt solution (0.1 M $\mathrm{NaCl}$ ) leaving the polyelectrolyte multilayers intact. By comparison an unmodified aluminium test piece revealed corrosion defects after just $12 \mathrm{~h}$ immersion in the same concentration of salt solution [30]. Three mechanisms of corrosion protection are observed: (i) passivation of the metal degradation by an inhibitor release;

(ii) buffering of $\mathrm{pH}$ changes at the corrosive area by polyelectrolyte layers; and

(iii) self-healing of the defects owing to the mobility of the polyelectrolyte components in the layer-by-layer assembly [31].

In a further investigation into the effectiveness of the self-healing action of coatings on aluminium specimens - the coating comprised a co-polymer consisting of polychlorotrifluoroethylene (PCTFE) and vinylether - a mass loss measurement was carried out after a $120 \mathrm{~h}$ immersion test in $3 \% \mathrm{NaCl}$ solution. The mass loss of the bare substrate was about $28 \mathrm{mg}$, whereas the mass loss of the coated product was about $1 \mathrm{mg}$ [32]. The main disadvantages of polymeric coatings, compared with those based on metals or ceramics, are poor mechanical properties, e.g. lower wear resistance or hardness, and a lack of electrical conduction. Reduced mechanical properties limit their applications. The improvement of some mechanical properties can be obtained by incorporation of nanomaterials but other properties may be deleteriously affected at the same time [33]. One of the essential issues is crack formation during the synthesis of polymer composite systems. The lack of electrical conduction can be overcome by the use of intrinsically conductive polymers (ICP) such as polypyrrole or polyaniline. Alternatively, polymeric matrices can be made to conduct electricity by incorporating conductive fillers (e.g. silver, gold, nickel, copper and carbon) in appropriate concentrations. In this way the performance of certain polymers can be significantly enhanced, leading to a broader range of potential applications. Carbon nanotubes (CNTs) are recognized as ideal conductive fillers because of their excellent electrical conductivity and nanoscopic size. Incorporated into electrically conductive epoxies 
(ECAs), carbon nanotubes additionally improve adhesion and mechanical properties (including strength) compared to unmodified epoxy [33].

\subsection{Capsule-based coatings}

The addition of micro- or nanocapsules, loaded with corrosion inhibitors, into coatings is another approach to achieving a protective coating with self-healing attributes. Capsules are typically constructed from polymers, inorganic materials like silica and titania and calcium carbonate [34-41]. Also halloysite aluminosilicate nanotubes are found as an entrapment system for storage of anticorrosion agents [42]. Micro- or nanocontainers can be inserted into a polymeric coating [34-37,41], a sol-gel coating [37-39,41-43], and occasionally into a metallic coating, e.g. one based on electrochemical nickel [40]. The self-repair properties of such coatings are activated by appropriate stimuli: temperature changes, radiation, $\mathrm{pH}$ changes, pressure changes and mechanical action [44]. However, not all mechanical damage is a consequence of the corrosion process and corrosion is not always initiated at coating's surface cracks. The most reliable and case-selective trigger for protective self-healing coatings containing capsules, is the change of electrochemical potential, which decreases when corrosion occurs [44].

The self-healing action of a coating containing encapsulated corrosion inhibitors, is contingent upon the formation of a passive layer on the underlying metal. Depending on the material being protected, this barrier layer can be produced by 2mercaptobenzothiazole, hexamethylene diisocyanate, linseed oil (for steel); $\mathrm{Ce}^{3+}$ compounds, silyl ester, alkoxysilanes, benzotriazole and 8-hydroxyquinoline (for AA 2024) [45-49]. The encapsulation of the corrosion inhibitors, instead of direct addition to the coating, is a viable way of avoiding potential problems such as coating integrity degradation, inhibitor deactivation or undesired leaching. The literature cites three independent studies into the durability of epoxy resin coatings incorporating encapsulated corrosion inhibitors with differing formulations. Improvement in the durability of the coatings was observed in each case and the results summarised below.

- A pure epoxy coating, immersed in $0.02 \mathrm{M} \mathrm{NaCl}$ solution, exhibited clearly visible corrosion products after 30 days (at room temperature). A water-borne 
epoxy coating with L-Carnosine (which is used as an inhibitor) contained within hollow mesoporous zirconia nanospheres (HMZSs) was unaffected after 10 days of immersion in $0.02 \mathrm{M}$ of $\mathrm{NaCl}$. The same water-borne epoxy coating with the zirconia nanospheres but in this case encapsulating corrosion inhibitor (nanocontainers constructed by directly binding L-CAR to HMZSs) provided a persistent barrier type of protection that was also unaffected after 30 days immersion [50].

- A plain epoxy coating on Q345 steel substrate, had delaminated after 27 days immersed in $3.5 \mathrm{wt} \% \mathrm{NaCl}$ solution. Whereas epoxy resin with 2-ercaptobenzothiazole (MBT)-intercalated Zn-Al-layered double hydroxides (LDHs) was unaffected after the same period of immersion in the salt solution. [51].

- An epoxy resin coating containing microcapsules with urea-formaldehyde as a shell and linseed oil as a healing agent, demonstrated a charge transfer resistance in $0.5 \mathrm{M} \mathrm{HCl} 1000$ times higher than a plain epoxy coating [52]. Additionally, the scratched area on the blank coating rapidly corroded with visible rust formation just after 60 min of exposure to corrosive environment, whereas the sample with coating containing microcapsules not only showed no visual evidence of corrosion, but also exhibited a self-healing behaviour.

The mechanism of the self-healing action in a coating containing capsules is presented in fig. 6 (Reprinted with permission from [53]).

Replacement of the capsules with microgels (micron-sized gel particles) is a new solution enabling the production of a container for an active self-healing compound much simpler than synthesising capsules. The manufacture of the microgel and incorporation of the active substances can now be achieved in one stage [6]. The production of capsules followed by the filling process clearly has a minimum of twostages. In the case of microgel preparation, the corrosion inhibitor can be added during its formation. An example of such a system is the preparation of polyurea microgel particles containing 2-methylbenzothiazole [6]. Differences between the physical construction of capsules and microgels are shown schematically in fig. 7.

2.3 Hybrid oxide coatings 
Hybrid oxide coatings, like thin functional siloxane-modified coatings, are employed as pre-treatment layers. They are considered as 'molecular bridges' and are used for increasing the bond strength between additional organic layers, such as paints and other adhesives, and the underlying substrate. Having both inorganic and organic groups, these materials can bond not only with the native oxide film on the metallic substrate but also with the uppermost paint system.

Siloxane compounds are largely inorganic in nature and exhibit resistance to most common ageing processes such as oxidation, corrosion, UV degradation, thermal gradients and mechanical stresses. Modifying polymeric matrices with Si-O has been shown to enhance their protective and barrier properties [54]. Siloxanes can be employed to modify a range of polymers such as epoxy, acrylates and polyurethanes. Acrylic-siloxanes are characterized by good weathering resistance, good barrier properties and corrosion resistance. Epoxy paints modified with siloxanes possess limited permeability for water and oxygen and good ionic resistance. These attributes are clearly beneficial in corrosion protection of metallic structures.

Hybrid oxide coatings can be modified to exhibit self-healing properties by the addition of corrosion inhibitors to the polymeric matrix. This is achieved by direct introduction of the chemicals or by encapsulating them first. To avoid the deterioration of their barrier properties the choice of an active substance should be made carefully. An example of a self-healing hybrid oxide coating utilising corrosion inhibitors and exhibiting increased durability is based on silicon and zircon oxides. Specifically it comprises a $\mathrm{SiO}_{x}-\mathrm{ZrO}_{x}$ hybrid film containing halloysite (aluminosilicate clay mineral) nanotubes filled with anticorrosive agents (benzotriazole and 8-hydroxyquinoline). Current-density measurements were carried out via scanning vibrating electrode technique (SVET). The anodic current in a plain coating monotonically increased after immersion in $0.1 \mathrm{M}$ $\mathrm{NaCl}$ for $3.5 \mathrm{~h}$ with a maximum value occurring after $10 \mathrm{~h}$. In contrast, the anodic current observed with the equivalent hybrid coating containing active compounds was reduced to noise level within $2 \mathrm{~h}$, being an evidence for inhibiting effect of benzotriazole on aluminium surface [55].

The corrosion protection properties of self-assembled nanophase particle (SNAP) coatings is also reported. Coatings are composed of organo-silane compounds doped with a corrosion inhibitor namely mercaptobenzimidazole (MBI), which is encapsulated 
with $\beta$-cyclodextrin in a dilute Harrison's solution (0.35 wt.\% $\left(\mathrm{NH}_{4}\right)_{2} \mathrm{SO}_{4}$ and 0.05 wt.\% $\mathrm{NaCl}$ ). The addition of the $\mathrm{MBI}$ to the hybrid organo-ceramic coating system demonstrated a significant improvement in corrosion protection of aluminium [56]. In the case of SNAP coatings containing MBI/ $\beta$-cyclodextrin complexes, no surface deterioration was observed after 8 days immersion in the corrosive electrolyte. Furthermore, compared with the undoped SNAP system, the coating containing MBI showed a fifty-fold decrease in the anodic corrosion current density during the 8 days immersion period [56].

\subsection{Other coatings}

In recent years, there have been many papers addressing other self-repair protective coatings for metallic structures. Examples of such systems are presented below:

- conversion coatings - the self-healing effect is achieved by forming an oxide or hydroxide layer enriched with elements from the coating and/or protected substrate [57];

- coatings containing fluoroorganic compounds $(\mathrm{COOH}, \mathrm{OH}, \mathrm{COF})$ - the mechanism for the self-healing ability of these coatings is based on the formation of a barrier film [58];

- coating based on titania and casein - depending on the $\mathrm{pH}$ casein forms agglomerates or is dispersed and the self-healing effect of the coating appears with the release of the casein and $\mathrm{TiO}_{2}$ particles in alkaline environment [59];

- ceramic materials $\left(\mathrm{TiC} / \mathrm{Al}_{2} \mathrm{O}_{3}\right.$ and $\mathrm{Ti}_{2} \mathrm{AlC}$ ) - the self-healing action is activated by an increase in temperature, causing the formation of titania and alumina [60];

- metallic coatings - the self-repair action depends on the type of polymer incorporated into them.

For example, the self-healing properties in zinc coating with polyethylene oxide-bpolystyrene ( $\mathrm{PEO}_{113-b}-\mathrm{PS}_{218}$ ) are based on an ability of amphiphilic polymers to shrink and swell reversibly [61]. The charge transfer resistance in $5 \% \mathrm{NaCl}$ solution of a coating reinforced with polymeric nanoaggregates is more than 7 times higher comparing to the charge transfer resistance of a pure zinc coating. A longer protection and increased service life for a steel substrate can be predicted. 
In the case of vanadia-based conversion coatings on magnesium alloys, after 7 days of immersion in $3.5 \% \mathrm{NaCl}$ solution, visual inspection of the plain and coated samples was carried out. The uncoated sample showed severe pitting and crevice corrosion with the average number of pits on the surface reaching about 50 within an area of 1 $\mathrm{cm}^{2}$. However, a noticeable decrease in the number and the size of the pits was observed for the sample treated with the vanadia conversion coating. In this instance, the number of pits in $1 \mathrm{~cm}^{2}$ was reduced to 2 [62].

The barrier and self-healing abilities of a corrosion-protective coating with a fluoroorganic compound for zinc were observed in $0.0005 \mathrm{M} \mathrm{NaCl}$ solution. After 7 days the scratched zinc specimen was covered by white rust, whilst the specimen coated with the fluoro-organic compound was largely unaffected with only a few observable surface pits [58].

\section{Patented solutions}

Among self-healing protective coatings patented to date, those inclusive capsules dominate. Microcapsules are formed with polymers or core/shell particles, like $\mathrm{CaCO}_{3}$ or halloysite [63-66]. The shell material for an inventive use can be also made of a naturally produced or occurred materials, like carbohydrate, glycoprotein, chitin or a lipid bilayer membrane [67]. Most often, microcapsules form part of the protective systems based on paints, whereas core/shell particles can be co-deposited with metallic coatings, such as nickel obtained by electrochemical means [68]. As a matrix for self-healing coatings sol-gel materials, organofunctional silane or polyelectrolytes are also used. Various compounds, in particular, organic -triazoles, quinolines, phosphonic acids, surfactants/amphiphiles, polymers, or inorganic -salts or solutions of salts such as oxides, hydroxides, carbonates, phosphates, are used as corrosion inhibitors [67].

A system described by Nippon Steel Corporation in 1994 is recognized as a forerunner for self-repair coatings containing microcapsules [69]. Authors patented a protective layer consisting zinc and additional fine or colloidal particles (oxides of silicon, titanium, chromium, aluminium, as well as zirconium). The corrosion resistant cores include water-soluble phosphates or molybdates. 
An interesting exemplar of patented self-healing coatings is solution based on a metallic microcapsule (built with nickel and zinc) that contains one or more polymeric precursors, as shown schematically in fig. 8. If the healing process provided by the resin is incomplete, the Ni:Zn spheres are sacrificed anodically in lieu of the steel substrate [70].

One of the main objectives of developing a self-healing, corrosion protective coating is to extend the service life of the metallic structure onto which it is applied. However, the lifetime of the underlying structure must also depend on the resilience of the coating. In which case it is pertinent to select some patented self-healing systems and consider their durability.

- The maximum lifetime of latex paint is typically ten years on exterior surfaces. On interior coatings, the maximum service life for this paint is four years. The use of enhanced coatings, which are made by addition of microcapsules built with polybutene or $\mathrm{Ca}(\mathrm{OH})_{2}$, as well as 50:50 mixture of both of them to latex paint, is projected to extend the coating lives by approximately 4.2-6 years for exterior coatings and by approximately 2.4 years for interior coatings [71].

- Epoxy coating containing a corrosion inhibitor of a barium salt of dinonylnaphthalene monosulfonic acid deposited on steel bars has shown no signs of corrosion in $3 \% \mathrm{NaCl}$ solution (temperature $65.5^{\circ} \mathrm{C}$ ) for 100 days. On the other hand, an epoxy coating without the active substance lasted only 14 days before the onset of corrosion of the sample steel bar [72].

- Another protective system included a cleansing coat, a self assembled monolayer phosphonate (SAMP) primer, a polyurethane primer containing zinc flake, and a polyurethane top coat. The top coat comprised self-healing diisocyanate/paraffin wax microcapsules and fixotropic additives (it can interchange between a solid state and a flowing liquid state according to prevailing conditions). The service life of the coating was extended by some $538 \%$ compared with certain other systems utilising polymers or ceramics [73].

\section{Commercially available solutions}


In spite of the commitment to undertake research into producing coatings with selfrepair properties by both industry and academia, there are still very few commercially available products. A product currently in development, is the Smart Corrosion Detector ${ }^{\circledR}$ offered by Battelle, a non-profit R \& D organization [74]. Battelle produced smart beads that not only detect corrosion but also provide a means of healing microscopic cracks on the substrate surface. The beads fluoresce in the presence of corrosion, fig. 9. However Battelle is still looking for collaborators to help extend the product applications.

A second system is the self-healing technology demonstrated by Autonomic Materials, Inc. [75]. Self-healing functionality is achieved in commercial thermosetting and elastomeric coatings by using two different polydimethylsiloxane (PDMS)-based chemistries. This coating has found an application in the industrial and marine sectors. Autonomic Materials offers a range of self-healing solutions compatible with the chemistry of most currently available coatings.

Another protective system, described as scratch and pinhole tolerant/self-healing, consists of a primer containing a unique conducting polymer nano dispersion (CPND), a top layer, and an optional interlayer. This coating technology system is called AnCatt and is employed as an effective barrier for protecting metals against corrosion without using any heavy-metal pigments such as chromate, lead, or zinc. Compared with current anti-corrosion coating products on the market, the AnCatt system offers 3-6x extension in longevity of the coating. This coating system was tested in accordance with ASTM B117 for 13,000 hours in a salt fog with no rusting or blistering of the substrate [76].

\section{Conclusions}

Extending the lifetimes of coatings to protect against corrosion by giving them selfhealing properties, has generated widespread academic and industrial interest. Nevertheless, commercially viable production of materials possessing ideal self-repair attributes is still some way off. For instance, considering organic polymers as a material for self-healing protective coatings, one can find few factors limiting their application, although susceptibility to weathering and ageing are still of concern. The fact remains that even coatings having a self-healing function can only slow down the degradation 
process. In the opinion of the authors, future developments should encompass the following:

- simplification of protective systems - the simpler the formulation and production methods, the broader the applicability;

- expansion of the contribution of natural materials to protective coatings - the additional benefit that derives from reducing environmental impact;

- development and application of siloxane technology - being a primer for the most often employed protective systems containing paint or polymer coatings as the top coat, functional silane coatings can effectively prolong their usefulness;

- investigation into metallic coatings - an opportunity to produce self-healing coatings with enhanced hardness and wear resistance.

\section{References}


[1] IOM3, http://www.iom3.org, 2015-01-30.

[2] V.S. Protsenko, F.I. Danilov, 2014, Chromium electroplating from trivalent chromium baths as an environmentally friendly alternative to hazardous hexavalent chromium baths: comparative study on advantages and disadvantages, Clean Technology and Environmental Policy, 16, 1201-1206.

[3] S.R. White, N.R. Sottos, P.H. Geubelle, J.S. Moore, M.R. Kessler, S.R. Sriram, E.N. Brown, S. Viswanathan, 2001, Autonomic healing of polymer composites, Nature, 409, 794-797.

[4] V. Karpakam, K. Kamaraj, S. Sathiyanarayanan, G. Venkatachari, S. Ramu, 2011. Electrosynthesis of polyaniline-molybdate coating on steel and its corrosion protection performance, Electrochimica Acta, 56, 2165-2173.

[5] R. Arefinia, A. Shojaei, H. Shariatpanahi, J. Neshati, 2012, Anticorrosion properties of smart coating based on polyaniline nanoparticles/epoxy-ester system, Progress in Organic Coatings, 75, 502-508.

[6] D. O. Grigoriev, K. Köhler, E. Skorb, D. G. Shchukin, H. Möhwald, 2009, Polyelectrolyte Complexes as "Smart" Depot for Self-Healing Anticorrosion Coatings, Soft Matter, 5, 1426-1432.

[7] T. F. Da Conceicao, N. Scharnag, W. Dietzel, D. Hoeche, K. U. Kainer, 2001, Study on the interface of PVDF coatings and HF-treated AZ31 magnesium alloy: Determination of interfacial interactions and reactions with self-healing properties, Corrosion Science, 53, 712-719.

[8] B. Aïssa, R. Nechache, E. Haddad, W. Jamroz, P. G. Merle, F. Rosei, 2012, Ruthenium Grubbs' catalyst nanostructures grown by UV-excimer-laser ablation for self-healing applications, Applied Surface Science, 258, 9800-9804.

[9] A. Yabuki, K. Okumura, 2012, Self-healing coatings using superabsorbent polymers for corrosion inhibition in carbon steel, Corrosion Science, 59, 258262.

[10] Z. Zhang, Y. Hu, Z. Liu, T. Guo, 2012, Synthesis and evaluation of a moisturepromoted healing copolymer, Polymer, 53, 2979-2990.

[11] D. Andreeva, D. Fix, H. Möhwald, D. G. Shchukin, 2008, Self-healing anticorrosion coatings based on $\mathrm{pH}$-sensitive polyelectrolyte/inhibitor sandwichlike nanostructures, Advanced Materials, 20, 2789-2794.

[12] M. Kendig, M. Hon, L. Warren, 2003, 'Smart' corrosion inhibiting coatings, Progress in Organic Coatings, 47, 183-189. 
[13] D. Y. Wu, S. Meure, D. Solomon, 2008, Self-healing polymeric materials: A review of recent developments, Progress in Polymer Science, 33, 479-522.

[14] A. Stankiewicz, I. Szczygieł, B. Szczygieł, 2013, Self-healing coatings in anticorrosion applications, Journal of Materials Science, 48, 8041-8051.

[15] X. Chen, F. Wudl, A. K. Mal, H. Shen, S. R. Nutt, 2003, New Thermally Remendable Highly Cross-Linked Polymeric Materials, Macromolecules, 36, 1802-807.

[16] P. Cordier, F. Tournilhac, C. Soulié-Ziakovic, L. Leibler, 2008, Self-healing and thermoreversible rubber from supramolecular assembly, Nature, 451, 977-980.

[17] S. J. Kalista, T. Ward, J. R. Soc, 2007, Thermal characteristics of the selfhealing response in poly(ethylene-co-methacrylic acid) copolymers, Interface, 4, 405-411.

[18] S. Burattini, H. M. Colquhoun, J. D. Fox, D. Friedmann, B. W. Greenland, P. J. F. Harris, W. Hayes, M. E. Mackay, S. J. Rowan, 2009, A self-repairing, supramolecular polymer system: healability as a consequence of donoracceptor $\pi-\pi$ stacking interactions, Chemical Communications, 6717-6719.

[19] X. Yan, F. Wang, B. Zheng, F. Huang, 2012, Stimuli-responsive supramolecular polymeric materials, Chemical Society Reviews, 41, 6042-6065.

[20] V. Kumar Thakur, M. R. Kessler, 2015, Self-healing polymer nanocomposite materials: A review, Polymer, 69, 369-383.

[21] C.B. Breslin, A.M. Fenelon, K.G. Conroy, 2005, Surface engineering: corrosion protection using conducting polymers, Materials and Design, 26, 233-237.

[22] T. Tüken, B. Yazıcı, M. Erbil, 2006, The corrosion behaviour of polypyrrole coating synthesized in phenylphosphonic acid solution, Applied Surface Science, 252, 2311-2318.

[23] P. P. Deshpande, N. Jadhav, V. J. Gelling, D. Sazou, 2014,Conducting polymers for corrosion protection: a review, Journal of Coatings Technology and Research, 11, 473-494.

[24] G. Williams, H. N. McMurray, 2005, Factors affecting acid-base stability of the interface between polyaniline emeraldine salt and oxide covered metal, Electrochemical and Solid-State Letters, 8, B42-B45.

[25] S. Radhakrishnan, C. R. Siju, D. Mahanta, S. Patil, G. Madras, 2009, Conducting polyaniline-nano- $\mathrm{TiO}_{2}$ composites for smart corrosion resistant coatings, Electrochimica Acta, 54, 1249-1254. 
[26] T. H. Tran, A. Vimalanandan, G. Genchev, J. Fickert, K. Landfester, D. Crespy, M. Rohwerder, 2015, Regenerative nano-hybrid coating tailored for autonomous corrosion protection, Advanced Materials, 27, 3825-3830.

[27] L. M. Baird, M. W. Patchan, M. Morris, A. J. Maisano, T. E. Phillips, J. J. Benkoski, R. Srinivasan, 2015, Synergy between galvanic protection and selfhealing paints, Langmuir, 31, 10610-10617.

[28] X. Luo, P. t. Mather, 2013, Shape memory assisted self-healing coating, Macro Letters, 2, 152-156.

[29] A. Yabuki, T. Nishisaka, 2011, Self-healing capability of porous polymer film with corrosion inhibitor inserted for corrosion protection, Corrosion Science, 53, 4118-4123.

[30] D. V. Andreeva, D. Fix, H. Möhwald, D. G. Shchukin, 2008, Buffering Polyelectrolyte Multilayers for Active Corrosion Protection, Journal of Material Chemistry, 18, 1738-1740.

[31] D. V. Andreeva, D. Fix, H. Möhwald, D. G. Shchukin, 2008, Self-healing anticorrosion coatings based on $\mathrm{pH}$-sensitive polyelectrolyte/inhibitor sandwichlike nanostructures, Advanced Materials, 20, 2789-2794.

[32] A. Yabuki, H. Yamagami, K. Noishiki, 2007, Barrier and self-healing abilities of corrosion protective polymer coatings and metal powders for aluminum alloys, Materials and Corrosion, 58, 497-501.

[33] B. M. Bailey, Y. Leterrier, S.J. Garcia, S. van der Zwaag, V. Michaud, 2015, Electrically conductive self-healing polymer composite coatings, Progress in Organic Coatings, 85, 189-198.

[34] X. Liu, H. Zhang, J. Wang, Z. Wang, S. Wang, 2012, Preparation of epoxy microcapsule based self-healing coatings and their behaviour, Surface and Coatings Technology, 206, 4976-4980.

[35] D. Snihirova, S. V. Lamaka, M. F. Montemor, 2012, "SMART" protective ability of water based epoxy coatings loaded with $\mathrm{CaCO}_{3}$ microbeads impregnated with corrosion inhibitors applied on AA2024 substrates, Electrochimica Acta, 83, 439-447.

[36] V. Sauvant-Moynot, S. Gonzalez, J. Kittel, 2008, Self-healing coatings: An alternative route for anticorrosion protection, Progress in Organic Coatings, 63, 307-315. 
[37] P. D. Tatiya, R. K. Hedaoo, P.P. Mahulikar, V. V. Gite, 2013, Novel Polyurea Microcapsules Using Dendritic Functional Monomer: Synthesis, Characterization and Its Use in Self-Healing and Anticorrosive Polyurethane Coatings, Industrial Engineering Chemistry Research, 52, 1562-1570.

[38] D. Borisova, H. Möhwald, D. G. Shchukin, 2013, Influence of Embedded Nanocontainers on the Efficiency of Active Anticorrosive Coatings for Aluminum Alloys Part II: Influence of Nanocontainer Position, ACS Applied Materials \& Interfaces, 5, 80-87.

[39] D. Borisova, H. Möhwald, D. G. Shchukin, 2011, Mesoporous Silica Nanoparticles for Active Corrosion Protection, ACS Nano, 5(3), 1939-1946.

[40] E. M. Moustafa, A. Dietz, T. Hochsattel, 2013, Manufacturing of nickel/nanocontainer composite coatings, Surface and Coating Technology, 216, 93-99.

[41] M. L. Zheludkevich, J. Tedim, M. G. S. Ferreira, 2012, "Smart" coatings for active corrosion protection based on multi-functional micro and nanocontainers, Electrochimica Acta, 82, 314-323.

[42] Y. M. Lvov, D. G. Shchukin, H. Möhwald, R. R. Price, 2008, Halloysite Clay Nanotybes for Controlled Release of Protective Agents, ACS Nano, 2, 814-820.

[43] M. L. Zheludkevich, D. G. Shchukin, K. A. Yasakau, H. Möhwald, M. G. S. Ferreira, 2007, Anticorrosion coatings with self-healing effect based on nanocontainers impregnated with corrosion inhibitor, Chemistry of Materials, 19, 402-411.

[44] A. Vimalanandan, L. Lv, T. Hai Tran, K. Landfester, D. Crespy, M. Rohwerder, 2013, Redox-Responsive Self-Healing for Corrosion Protection, Advanced Materials, 25, 6980-6984.

[45] N. Selvakumar, K. Jeyasubramanian, R. Sharmila, 2012, Smart coating for corrosion protection by adopting nano particles, Progress in Organic Coatings, 74, 461-469.

[46] M. Kouhi, A. Mohebbi, M. Mirzaei, 2012, Evaluation of the corrosion inhibition effect of micro/nanocapsulated polymeric coatings: a comparative study by use of EIS and Tafel experiments and the area under the Bode plot, Research on Chemical Intermediates, 39, 2049-2062. 
[47] X. Jiang, Y. Jiang, N. Liu, H. Xu, S. Rathod, P. Shah, C. F. Binker, 2011, Controlled release from core-shell nanoporous silica particles for corrosion inhibition of aluminum alloys, Journal of Nanomaterials, 2011, 1-10.

[48] M. Zheludkevich, R. Serra, M. Montemor, M. Ferreira, 2005, Oxide nanoparticle reservoirs for storage and prolonged release of the corrosion inhibitors, Electrochemistry Communications, 7, 836-840.

[49] S. V. Lamaka, M. L. Zheludkevich, K. A. Yasakau, R. Serra, S. K. Poznyak, M. G. S. Ferreira, 2007, Nanoporous titania interlayer as reservoir of corrosion inhibitors for coatings with self-healing ability, Progress in Organic Coatings, 58, 127-135.

[50] M. Wang, M. Liu, J. Fu, 2015, An intelligent anticorrosion coating based on pHresponsive smart nanocontainers fabricated via a facile method for protection of carbon steel, Journal of Materials Chemistry A, 3, 6423-6431.

[51] Y. Dong, F. Wang, Q. Zhou, 2014, Protective behaviors of 2mercaptobenzothiazole intercalated Zn-Al-layered double hydroxide coating, Journal of Coatings Technology and Research, 11, 793-803.

[52] M. Hasanzadeh, M. Shehidi, M. Kazemipour, 2015, Application of EIS and EN techniques to investigate the self-healing ability of coatings based on microcapsules filled with linseed oil and $\mathrm{CeO}_{2}$ nanoparticles, Progress in Organic Coatings, 80, 106-119.

[53] S. H. Cho, H. M. Andersson, S. R. White, N. R. Sottos, P. V. Braun, 2006, Polydimethylsiloxane-Based Self-Healing Materials, Advanced Materials,18, 997-1000.

[54] M.F. Montemor, 2014, Functional and smart coatings for corrosion protection: A review of recent advances, Surface and Coatings Technology, 258, 17-37.

[55] D. Fix, D. V. Andreeva, Y. M. Lvov, D. G. Shchukin, H. Möhwald, 2009, Application of inhibitor loaded halloysite nanotubes in active anti-corrosive coatings, Advanced Functional Materials, 19, 1720-1727.

[56] A.N. Khramov, N.N. Voevodin, V.N. Balbyshev, M.S. Donley, 2004, Hybrid organo-ceramic corrosion protection coatings with encapsulated organic corrosion inhibitors, Thin Solid Films, 447 - 448, 549-557.

[57] K. Aramaki, 2003, Self-healing mechanism of a protective film prepared on a $\mathrm{Ce}\left(\mathrm{NO}_{3}\right)_{3}$-pretreated zinc electrode by modification with $\mathrm{Zn}\left(\mathrm{NO}_{3}\right)_{2}$ and $\mathrm{Na}_{3} \mathrm{PO}_{4}$ Corrosion Science, 45, 1085-1101. 
[58] A. Yabuki, R. Kaneda, 2009, Barrier and self-healing coating with fluoro-organic compound for zinc, Materials and Corrosion, 60, 444-449.

[59] A. Yabuki, M. Sakai, 2011, Self-healing coatings of inorganic particles using a pH-sensitive organic agent, Corrosion Science, 53, 829-833.

[60] J. Gao, J. Suo, 2011, Effects of heating temperature and duration on the microstructure and properties of the self-healing coatings, Surface and Coatings Technology, 206, 1342-1350.

[61] D. A. Koleva, N. Boshkov, V. Bachvarov, H. Zhann, J. H. W. de Wit, K. van Breugel, 2010, Application of $\mathrm{PEO}_{113}-\mathrm{b}-\mathrm{PS}_{218}$ nano-aggregates for improved protective characteristics of composite zinc coatings in chloride-containing environment, Surface and Coatings Technology, 204, 3760-3772.

[62] A. S. Hamdy, I. Doench, H. Möhwald, 2011, Assessment of a one-step intelligent self-healing vanadia protective coatings for magnesium alloys in corrosive media, Electrochimica Acta, 56, 2493-2502.

[63] C. Schmidt, Anti-corrosion coating for metals has at least one layer, e.g. electrophoretic primer, containing hollow micro-particles, some filled with corrosion inhibitors and some filled with isocyanate or other hardenable substance, DE10058595, 2002-06-06.

[64] J. Kron, Schichtsystem zum Korrosionsschutz, DE102012209761, 2013-12-12.

[65] T. Kumar Rout, Anti-corrosion system for steel, WO2013083293, 2013-06-13.

[66] P. Braun, S. Cho, S. White, Self-healing coating system, US20070166542, 2007-07-19.

[67] I. Grunwald, Composites and coating materials with active substances contained in biological enveloping material, WO2013083693 A1, 2013-06-13.

[68] A. Dietz, Layer system for corrosion protection consisting of substrate and coating containing core/shell particles useful in automobile industry and for internal combustion engines, DE1020004010212, 2005-09-22.

[69] T. Izaki, Corrosion-resistant plated composite steel strip and method of producing same, EP0323756, 1994-09-07.

[70] J. Benkoski, R. Srinivasan, J. Marandi, Self-Healing Coatings, US20110293958, 2011-12-01.

[71] A. Kumar, L. Stephenson, Self-healing coatings using microcapsules, US20060042504 A1, 2006-03-02. 
[72] C. R. Guilbert, D. G. Enos. C. E. Boyer, Latent coating for metal surface repair, WO1999046338A1, 1999-09-16.

[73] J. Jackson, M. Henry, Nano-based self-healing anti-corrosion coating, WO2012178193, 2012-12-27.

[74] http://www.battelle.org/media/press-releases/battelle-develops-self-healingsmart-corrosion-beads\#sthash.VbaTazu3.dpuf, 2015-07-10.

[75] http://www.autonomicmaterials.com/technologyl, 2015-07-10.

[76] http://www.ancatt.com/Anti Corrosion Coating.html, 2015-11-21.

Table 1 Specific examples of the self-healing mechanisms observed in polymeric systems

\begin{tabular}{|l|l|c|}
\hline \multicolumn{1}{|c|}{ Composition of coating } & \multicolumn{1}{|c|}{ Self-healing mechanism } & Ref. \\
\hline $\begin{array}{l}\text { 5-ethylidene-2- } \\
\text { norbornene matrix with a } \\
\text { Ruthenium Grubb's } \\
\text { Catalyst }\end{array}$ & $\begin{array}{l}\text { The self-healing mechanism is based on a } \\
\text { ring-opening metathesis polymerization } \\
\text { (ROMP). }\end{array}$ & [8] \\
\hline Conductive polymers & $\begin{array}{l}\text { 1. The oxidizing property of the conductive } \\
\text { polymer promotes passivation of the steel } \\
\text { substrate. } \\
\text { 2. When the conductive polymer is doped } \\
\text { with inhibitors the reduction of oxygen is } \\
\text { prevented. }\end{array}$ & $\begin{array}{c}\text { [12,21- } \\
23]\end{array}$ \\
\hline $\begin{array}{l}\text { Polyvinyl butyral with } \\
\text { nano-TiO } 2 \text {-polyaniline }\end{array}$ & $\begin{array}{l}\text { In this system three self-healing mechanisms } \\
\text { are recognized. } \\
\text { 1. Barrier properties. } \\
\text { 2. Redox behaviour of polyaniline. }\end{array}$ & {$[25]$} \\
\hline
\end{tabular}




\begin{tabular}{|l|l|l|}
\hline & $\begin{array}{l}\text { 3. Formation of p-n junctions preventing } \\
\text { charge transport after coating damage. }\end{array}$ & \\
\hline $\begin{array}{l}\text { Bilayer coating: zinc } \\
\text { coating and polyvinyl } \\
\text { butyral coating with } \\
\text { nanocapsules }\end{array}$ & $\begin{array}{l}\text { The self-healing mechanism is based on a } \\
\text { ring-opening metathesis polymerization } \\
\text { (ROMP). As a trigger to release the self- } \\
\text { healing agent and catalyst, a change in pH is } \\
\text { employed. }\end{array}$ & [26] \\
\hline $\begin{array}{l}\text { Paints with silanes and } \\
\text { zinc }\end{array}$ & $\begin{array}{l}\text { The zinc-based cathodic protection of steel is } \\
\text { enhanced by polarizing steel with silanes } \\
\text { Additionally the rate of zinc oxidation is } \\
\text { lowered by silanes thereby extending the } \\
\text { lifetime of galvanic protection. }\end{array}$ & [27] \\
$\begin{array}{l}\text { A schematic representation of the process is } \\
\text { shown in Fig.4. }\end{array}$ & \\
\hline $\begin{array}{l}\text { Thermoplastic poly(ع- } \\
\text { caprolactone) (PLC) } \\
\text { fibres in a shape memory } \\
\text { epoxy matrix }\end{array}$ & $\begin{array}{l}\text { Heating triggers the shape recovery of the } \\
\text { matrix to bring the crack surfaces into close } \\
\text { proximity where the PLC fibres melt and } \\
\text { repair the crack. } \\
\text { Fig. } 5 \text { illustrates this process. }\end{array}$ & [28] \\
\hline
\end{tabular}

\section{Figure captions}

Fig.1 Number of publications on self-healing protective coatings in years 1984-2014, according to data provided by Scopus.

Fig.2 Global contribution to research on self-healing protective coatings, according to data provided by Scopus.

Fig.3 Contribution of particular countries to research on self-healing protective coatings in Europe, according to data provided by Scopus.

Fig.4 Paints based on the Chemical Agent Resistant Coatings (CARC) specification with a zinc layer enriched in microcapsules filled with octadecyltrimethoxysilane: 
(A) Standard CARC, (B) Zn-rich CARC, and (C) standard CARC with microcapsules, and (D) Zn-rich CARC with microcapsules.

Reprinted with permission from Ref. [27]. Copyright 2015 American Chemical Society.

Fig.5 Schematic illustration of the coating morphology and the shape memory assisted self-healing concept.

Reprinted with permission from Ref. [28]. Copyright 2013 American Chemical Society.

Fig.6 Schematic of the self-healing process: a) self-healing composite consisting of a microencapsulated catalyst (yellow) and phase-separated healing-agent droplets (white) dispersed in a matrix (green); b) crack propagating into the matrix, releasing the catalyst and healing agent into the crack plane; c) a crack healed by polymerized PDMS (crack width exaggerated). Scanning electron microscopy (SEM) images of d) the fracture surface, showing an empty microcapsule and voids left by the phase-separated healing agent, and e) a representative microcapsule showing its smooth, uniform surface.

Reprinted with permission from Ref. [53]. Copyright 2015 John Wiley and Sons.

Fig.7 Schematic illustration of a microgel and a capsule.

Fig.8 Microcapsule with double shell built with metals and polymers.

Fig. 9 The smart coating fluoresces in cracks under UV light. Reprinted with permission from Battelle. 


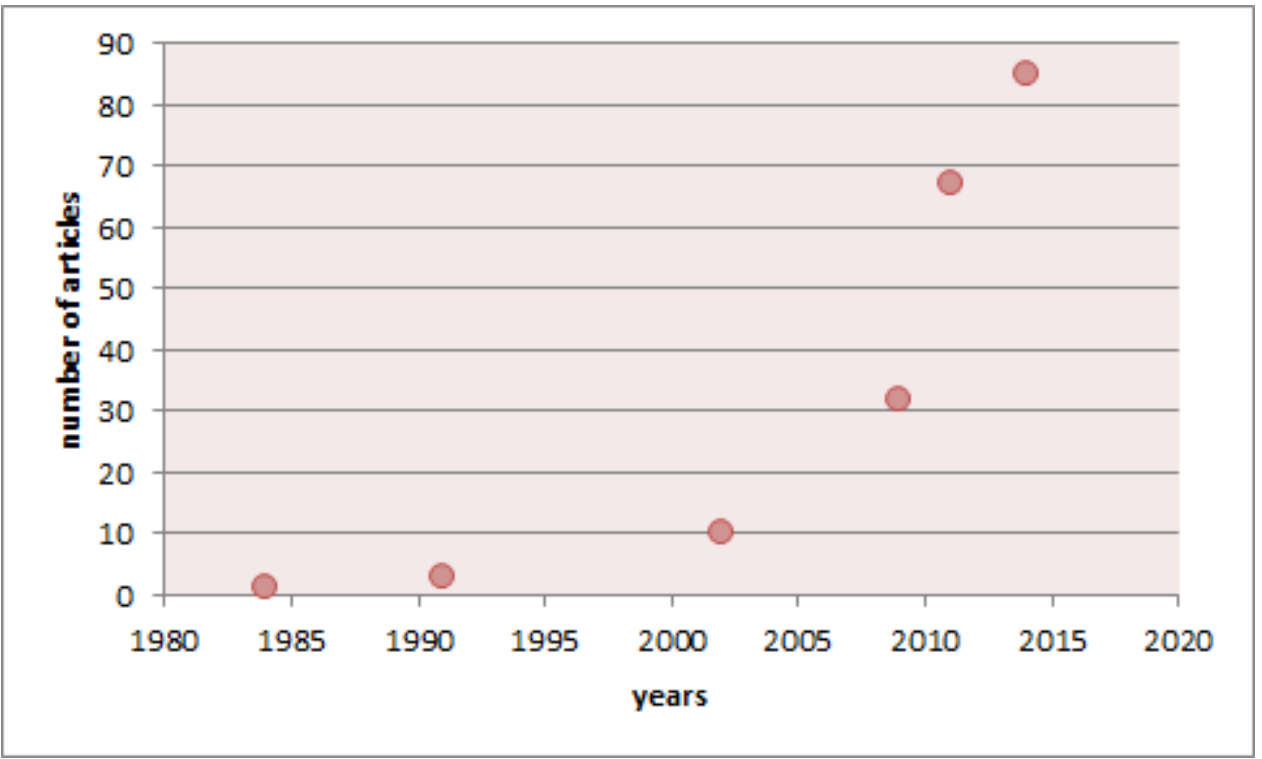

Fig. 1

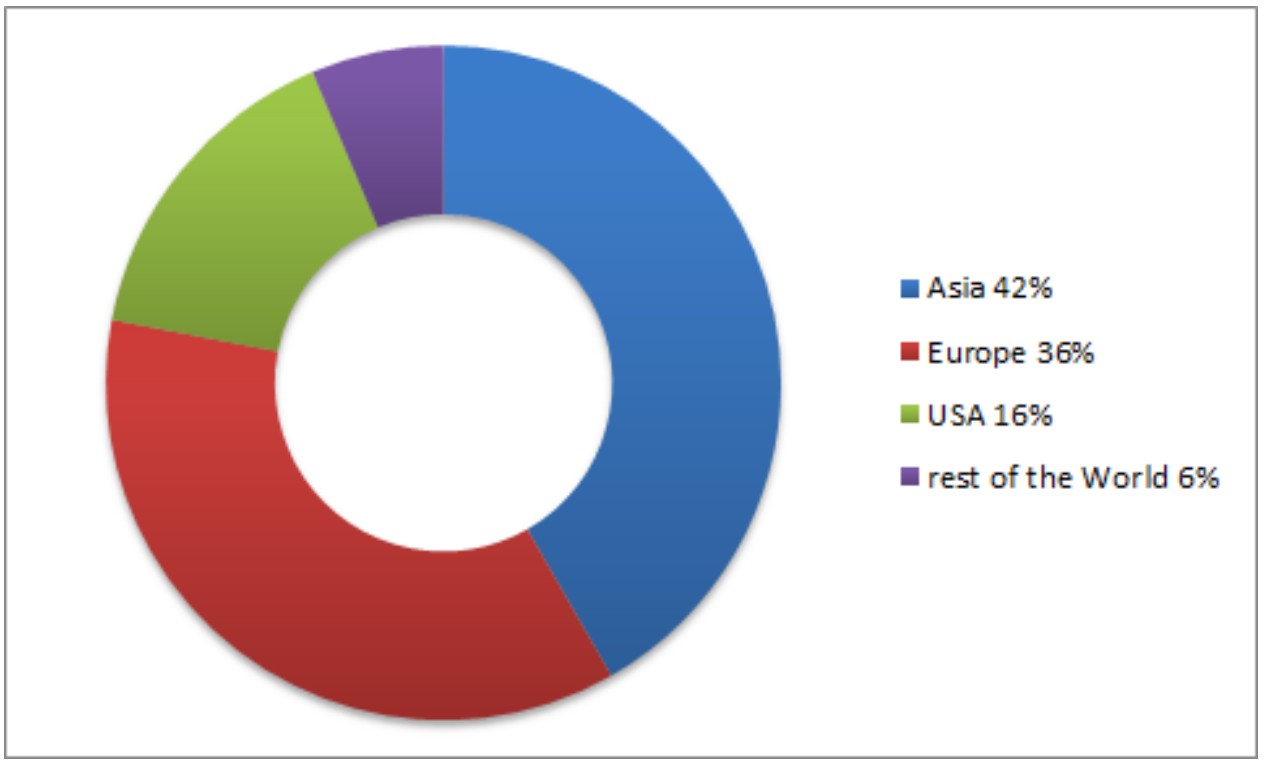

Fig. 2 


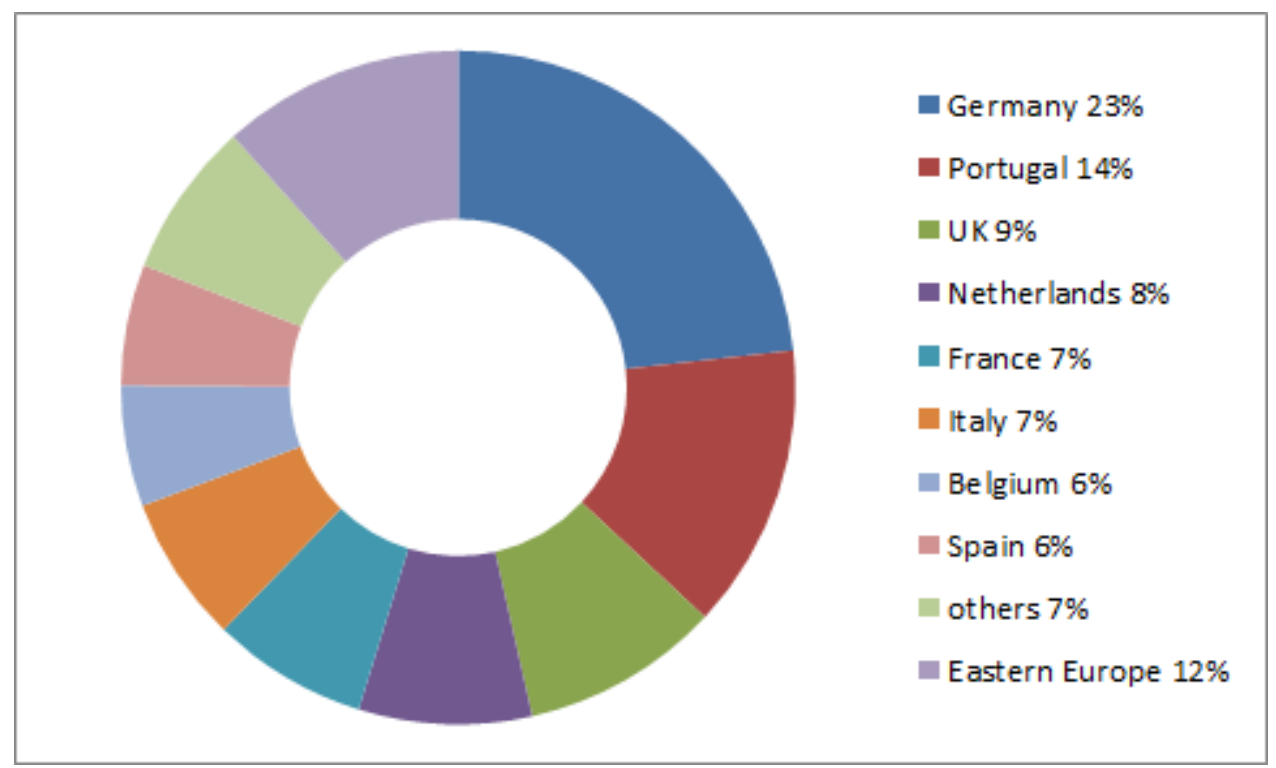

Fig. 3

ACS Publications Werg High quality Hightimpact

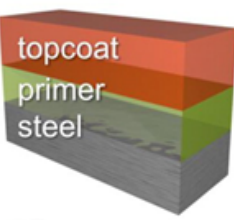

A)

topcoat
primer
sn-rich
steel
B)

C)

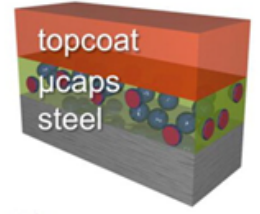

D

Published in: Lance Michael Baird; Marcia W. Patchan; Melanie Morris; Adam J. Maisano; Terry E. Phillips; Jason J. Benkoski; Rengaswamy Srinivasan: Langmuir 2015, 31,10610-10617.

DOI: 10.1021/acs.langmuir.5b02115

Copyright @ 2015 American Chemical Society

Fig. 4 


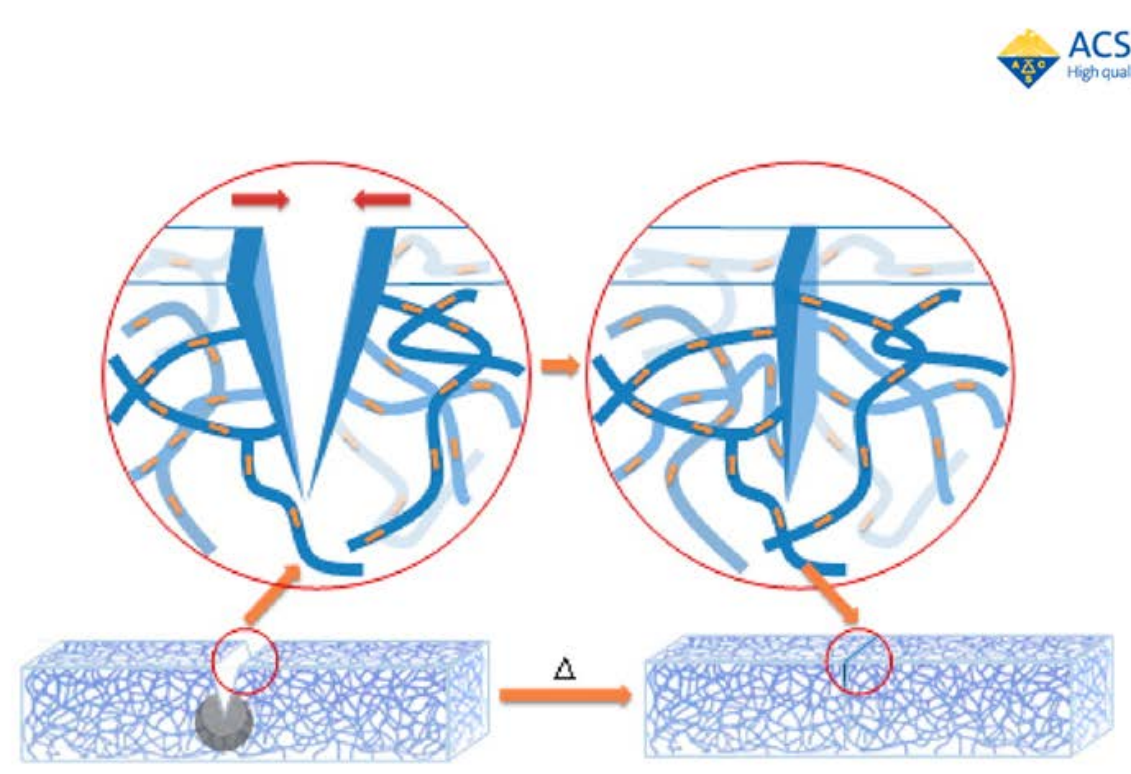

Published in: Xiaofan Luo; Patrick T. Mather; ACS Macro Lett. 2013, 2, 152-156.

Copyright @ 2013 American Chemical Society

Fig. 5

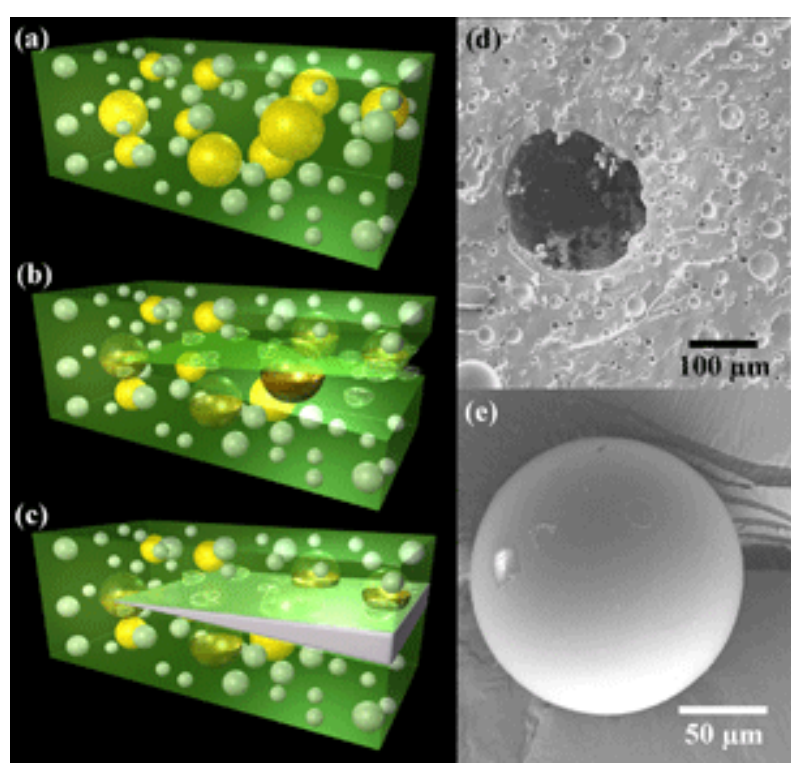

Fig. 6 


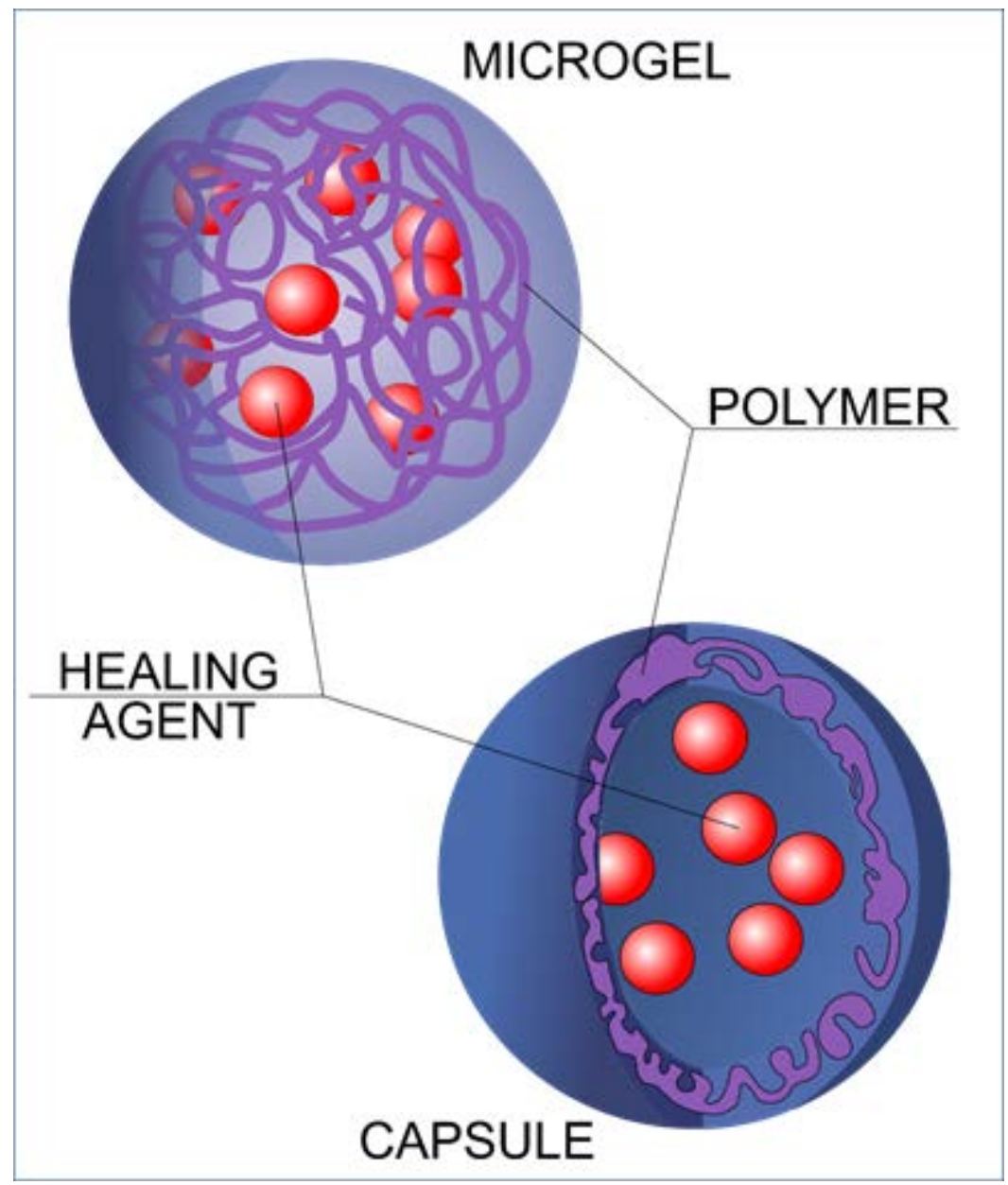

Fig. 7

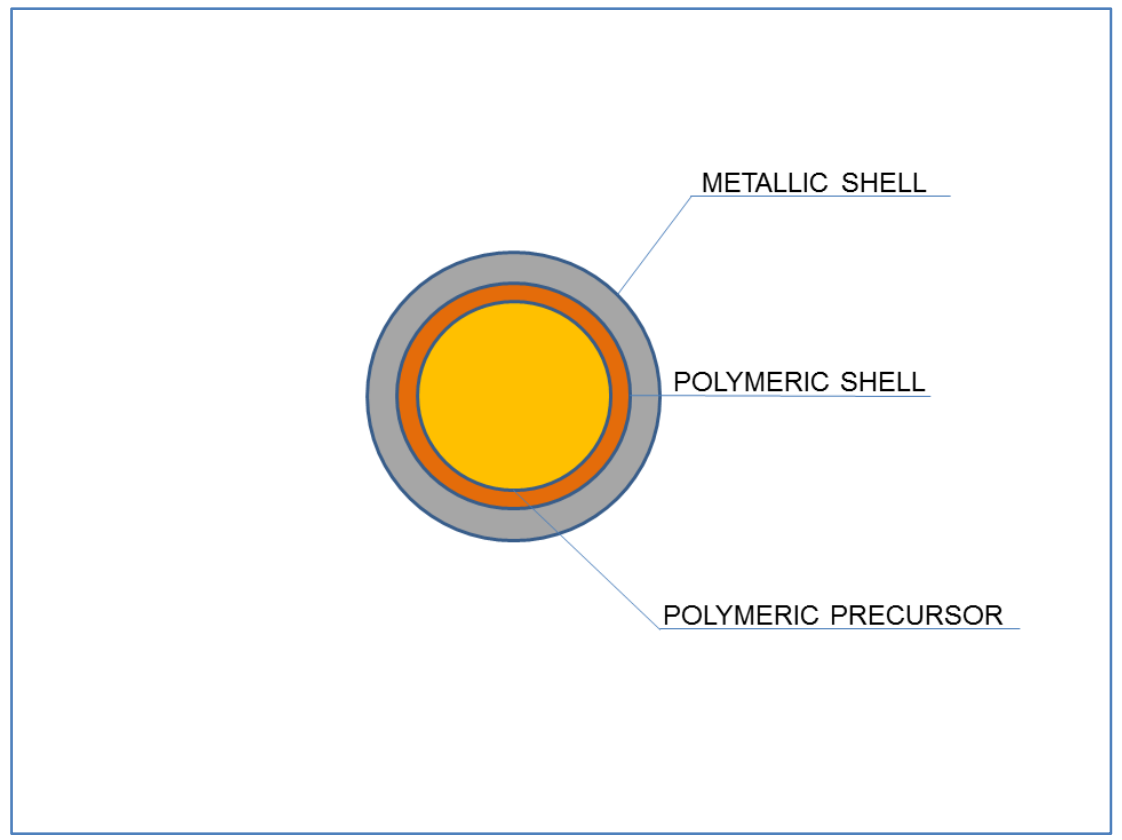

Fig. 8 


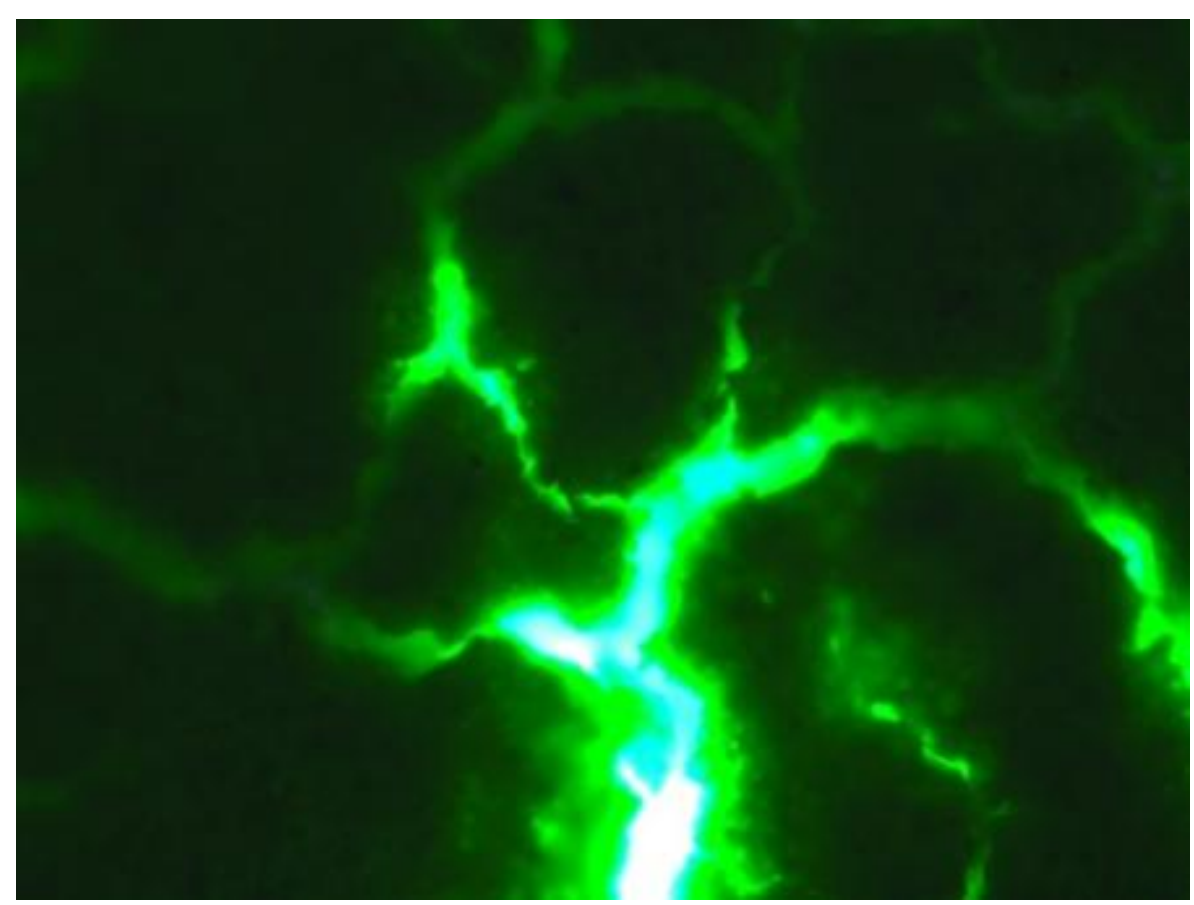

Fig. 9 